\title{
0175 ETHNIC DIFFERENCES IN INTIMATE PARTNER VIOLENCE BETWEEN MESTIZO AND INDIGENOUS WOMEN IN ECUADOR
}

M I Roldos* Correspondence: College of Public Health, University of Georgia, 1271 Sanden Ferry Drive, Decatur, GA 30033, USA

10.1136/ip.2010.029215.175

Intimate partner violence (IPV) is a pervasive socially learnt behaviour affecting close to one third of all women in Ecuador. Research done in other countries suggests that after controlling for socioeconomic circumstances and alcohol use, significant ethnic differences exist among minorities including higher crude rates of violence. This study analyzed a 2004 cross-sectional database on maternal and infant health (ENDEMAIN) that surveyed a stratified random sample of Ecuadorian Mestizo and Indigenous women between 15 and 49 years of age. Using a chi-square and a proportion t-test, we tested for significant ethnic differences in their self-report experience of IPV in the last 12 months and help-seeking behaviour. Results suggest no significant difference in the prevalence of IPV between Indigenous and Mestizo, even after controlling for income. However, for the $2 \%$ of women reporting seeking help after an episode of violence, her family was the most frequent place to seek help and the Women and Family Commissaries was the second one ( $p>.005)$. These findings suggest that IPV is a significant public health problem for all Ecuadorian women regardless of ethnicity. In addition, understanding ethnic differences of how and where women seek help will allow a more efficient allocation of resources to treat and prevent IPV. 